\title{
Update on intrathecal management of cerebral vasospasm: a systematic review and meta-analysis
}

\author{
Audrey A. Grossen, BA, Griffin L. Ernst, MD, and Andrew M. Bauer, MD \\ Department of Neurosurgery, University of Oklahoma Health Sciences Center, Oklahoma City, Oklahoma
}

OBJECTIVE Aneurysmal subarachnoid hemorrhage (aSAH) accounts for a relatively small portion of strokes but has the potential to cause permanent neurological deficits. Vasospasm with delayed ischemic neurological deficit is thought to be responsible for much of the morbidity associated with aSAH. This has illuminated some treatment options that have the potential to target specific components of the vasospasm cascade. Intrathecal management via lumbar drain (LD) or external ventricular drain (EVD) offers unique advantages in this patient population. The aim of this review was to provide an update on intrathecal vasospasm treatments, emphasizing the need for larger-scale trials and updated protocols using data-driven evidence.

METHODS A search of PubMed, Ovid MEDLINE, and Cochrane databases included the search terms (subarachnoid hemorrhage) AND (vasospasm OR delayed cerebral ischemia) AND (intrathecal OR intraventricular OR lumbar drain OR lumbar catheter) for 2010 to the present. Next, a meta-analysis was performed of select therapeutic regimens. The primary endpoints of analysis were vasospasm, delayed cerebral ischemia (DCI), cerebral infarction, and functional outcome.

RESULTS Twenty-nine studies were included in the analysis. There were 10 studies in which CSF drainage was the primary experimental group. Calcium channel antagonists were the focus of 7 studies. Fibrinolytics and other vasodilators were each examined in 6 studies. The meta-analysis included studies examining CSF drainage via LD $(n=4)$, tissue plasminogen activator in addition to $\operatorname{EVD}(n=3)$, intraventricular nimodipine $(n=2)$, and cisternal magnesium $(n=2)$. Results showed that intraventricular nimodipine decreased vasospasm (OR 0.59, 95\% Cl 0.37-0.94; $p=0.03$ ). Therapies that significantly reduced $\mathrm{DCl}$ were CSF drainage via LD (OR $0.47,95 \% \mathrm{Cl} 0.25-0.88 ; p=0.02$ ) and cisternal magnesium (OR $0.27,95 \% \mathrm{Cl} 0.07-1.02 ; p=0.05)$. CSF drainage via LD was also found to significantly reduce the incidence of cerebral infarction (OR 0.35, 95\% 0.24-0.51; $p<0.001)$. Lastly, functional outcome was significantly better in patients who received CSF drainage via LD (OR 2.42, 95\% Cl 1.39-4.21; $p=0.002)$.

CONCLUSIONS The authors' results showed that intrathecal therapy is a safe and feasible option following aSAH. It has been shown to attenuate cerebral vasospasm, reduce the incidence of $\mathrm{DCl}$, and improve clinical outcome. The authors support the use of intrathecal management in the prevention and rescue management of cerebral vasospasm. More randomized controlled trials are warranted to determine the best combination of pharmaceutical agents and administration route in order to formulate a standardized treatment approach.

https://thejns.org/doi/abs/10.3171/2021.12.FOCUS21629

KEYWORDS subarachnoid hemorrhage; vasospasm; intrathecal; intraventricular; lumbar drain; external ventricular drain

$\mathrm{S}$ UBARACHNOID hemorrhage is most commonly the result of traumatic injury, with the remaining majority of cases due to ruptured intracranial aneurysm. ${ }^{1}$ While aneurysmal subarachnoid hemorrhage (aSAH) accounts for a relatively small portion of strokes (approximately 5\%), it comes with high morbidity and mortality. Possible sequelae include hydrocephalus, vasospasm, delayed cerebral ischemia (DCI), cerebral infarction, and death.

In accordance with the uniform definitions outlined by
Vergouwen et al., ${ }^{2}$ vasospasm is an arterial narrowing after hemorrhage demonstrated on angiography or transcranial Doppler (TCD) ultrasonography. This prolonged arterial constriction is most likely to occur within the first 3 to 14 days following hemorrhage. However, the vasospasm period is considered to extend until 21 days after the initial hemorrhage. While the pathogenesis of vasospasm has yet to be fully elucidated, the mechanism involves early brain injury and subsequent inflammatory cascade. Prolonged vasoconstriction occurs secondary to irritation due

ABBREVIATIONS aSAH = aneurysmal subarachnoid hemorrhage; $\mathrm{DCl}=$ delayed cerebral ischemia; EVD = external ventricular drain; GOS = Glasgow Outcome Scale; $\mathrm{ICP}=$ intracranial pressure; $\mathrm{LD}=$ lumbar drain; $\mathrm{mRS}=$ modified Rankin Scale; $\mathrm{TCD}=$ transcranial Doppler; tPA = tissue plasminogen activator.

SUBMITTED November 1, 2021. ACCEPTED December 22, 2021.

INCLUDE WHEN CITING DOI: 10.3171/2021.12.FOCUS21629. 
to blood clotting and inflammatory mediators surrounding the vessel. Some of the key mediators include nitric oxide and endothelin-1, which induce oxidative stress and release free radicals surrounding the calcium channels of vascular smooth-muscle cells. ${ }^{3}$ Reports have estimated that the likelihood of vasospasm following aSAH ranges between $50 \%$ and $90 \%{ }^{4}$

DCI is defined as new neurological deficits such as decreased level of consciousness, aphasia, hemiparesis, or neglect. ${ }^{2}$ In the past, DCI has been attributed to vasospasm; however, past interventions have not been met with resounding results, suggesting that vasospasm is not the sole cause of DCI. Mechanisms, including blood-brain barrier disruption, microthrombosis, cortical spreading depolarizations, and failure of cerebral autoregulation, have also been suggested to play a role. ${ }^{4}$ DCI has a clear association with subsequent cerebral infarction and is one of the most common, and perhaps most preventable, causes of late neurological morbidity in this patient population.

Identifying patients at greatest risk of DCI is essential in preventing neurological and functional decline. Methods that have been historically used, in part, to prognosticate aSAH include the Hunt and Hess grade and World Federation of Neurosurgical Societies grading system. In recent years, there has been an emergence of new clinical prediction models that used pooled patient information from data sets, registries, and clinical trials. ${ }^{5}$ Additionally, computational fluid dynamics is currently being studied as a novel method to predict DCI before aneurysm treatment. ${ }^{6}$

Treatment of vasospasm can be divided into prophylaxis and rescue management. Prophylaxis is administered in the acute period after aSAH, before the occurrence of vasospasm. Rescue management is administered after radiographic or clinical evidence of vasospasm. Currently, the only medication approved by the US FDA that has been shown systematically to improve outcomes from aSAH is the calcium channel blocker nimodipine.

The traditional treatment for DCI was "triple-H" therapy involving induced hypertension, hypervolemia, and hemodilution. However, treatment protocols have shifted after increased morbidity developed from anemia, hyponatremia, a hypervolemic state, and complications from vasopressors. Most protocols currently include fluid repletion to maintain a euvolemic state while achieving a permissively elevated systolic blood pressure. Other common practices include placement of an external ventricular drain (EVD) to combat increased intracranial pressure (ICP) as well as avoidance of anemia and hyponatremia. ${ }^{3}$

New research continues to emerge regarding the pathogenesis of vasospasm. This has illuminated some treatment options that have the potential to target specific components of the pathology cascade. Our review provides an update on intrathecal vasospasm treatments, emphasizing the need for larger-scale trials and updated protocols using data-driven evidence.

\section{Methods}

\section{Systematic Review}

Eligibility Criteria

A systematic review of studies involving intrathecal therapeutic options for prevention and rescue management of vasospasm following aSAH was performed according to PRISMA guidelines. Articles were reviewed by one team member (A.A.G.), and interpretation was verified by a second team member (G.L.E.).

A search of the PubMed, Ovid MEDLINE, and Cochrane databases was conducted for the time period from 2010 to August 13, 2021, with the following search terms: (subarachnoid hemorrhage) AND (vasospasm OR delayed cerebral ischemia) AND (intrathecal OR intraventricular OR lumbar drain OR lumbar catheter).

Studies were included if they 1) included patients with confirmed aSAH; 2) investigated prophylactic or rescue therapy administered intrathecally, intracisternally, or intraventricularly for the management of vasospasm; and 3) presented original research. Animal studies and study protocols with no reported data were excluded from analysis. Reviews, meta-analyses, commentary, letters to the editor, editorials, and articles not accessible in the English language were also excluded.

\section{Data Extraction}

The following data points were extracted from each study: therapeutic agent, mode of administration, dosage, and patient population and control. Each data point was extracted, reviewed, and agreed on by the two reviewers. The primary outcomes of our analysis were vasospasm, DCI, cerebral infarction, and functional outcome.

\section{Meta-Analysis \\ Eligibility Criteria}

We conducted a meta-analysis of eligible studies from each therapeutic category (CSF drainage, fibrinolysis, calcium channel antagonists, and other vasodilators). Study inclusion criteria were as follows: 1) An intrathecal experimental group and a nonintrathecal control were included. 2) The therapeutic regimen and control were utilized by more than one study. 3) The incidence of vasospasm, DCI, cerebral infarction, or functional outcome was reported for both groups.

\section{Data Extraction}

Each eligible therapeutic regimen was identified and analyzed. Primary endpoints of analysis were vasospasm, DCI, cerebral infarction, and functional outcome. Analysis was conducted using Review Manager version 5.4.1 (The Cochrane Collaboration). Statistical significance was defined as $\mathrm{p} \leq 0.05$.

\section{Definitions}

Vasospasm was defined as arterial narrowing proven by angiography or TCD ultrasonography, and DCI was defined as new neurological deficits (e.g., decreased level of consciousness, aphasia, hemiparesis, or neglect). Cerebral infarction was identified on CT or MRI, or proven at autopsy. Complications were defined as adverse events following aSAH management, including hydrocephalus, hemorrhage or hematoma, pneumonia, meningitis, ventriculitis, angioplasty, heart failure, requiring a ventriculoperitoneal shunt, and septic shock. Functional outcome 
was determined by Glasgow Coma Scale, Glasgow Outcome Scale (GOS), extended GOS, or modified Rankin Scale (mRS) scores, whereby favorable outcome was reported as a Glasgow Coma Scale score of 13 to 15, a GOS score of 3 to 5 , an extended GOS score of 6 to 8 , or an $\mathrm{mRS}$ score of 0 to 3 at the longest follow-up.

\section{Results}

Search criteria yielded 256 unique results. Of these, 29 met our criteria and were included in the analysis (Fig. 1). Studies are grouped by therapeutic modality: CSF drainage, fibrinolysis, calcium channel antagonists, and other vasodilators (Tables $1-4)$.

Ten studies involved CSF drainage via either LD $(n=$ 8) or EVD $(n=1)$ (Table 1). The final study compared the use of a lumbar catheter with EVD. Of these studies, 1 showed a significant decrease in vasospasm, 3 showed significant decreases in DCI, 3 demonstrated significantly reduced cerebral infarction, and 5 had improved clinical outcomes. Fibrinolytic therapy was examined in 6 of the studies (Table 2). The experimental agent was tissue plasminogen activator (tPA) in 4 studies and urokinase in the remaining 2. Vasospasm, cerebral infarction, and clinical outcome were improved in 1 study each. None were shown to significantly decrease the incidence of DCI. Seven studies pertained to the intrathecal calcium channel antagonists nicardipine $(\mathrm{n}=5)$ and nimodipine $(\mathrm{n}=$ 2) (Table 3). While 4 of these studies showed significant improvement in vasospasm, none showed a decreased incidence of DCI, cerebral infarction, or improved clinical outcome. Six studies involved other vasodilators (Table 4), including milrinone $(\mathrm{n}=2)$, magnesium $(\mathrm{n}=2)$, and nitric oxide $(n=2)$. Three of these studies showed attenuation of vasospasm, 2 showed decreased DCI, 1 showed reduced incidence of cerebral infarction, and 1 showed improved clinical outcomes.

Therapeutic regimens included in meta-analysis were CSF drainage via a lumbar drain (LD) versus no $\mathrm{LD}(\mathrm{n}=$ 4), tPA+EVD versus EVD alone $(n=3)$, intraventricular nimodipine versus oral nimodipine $(\mathrm{n}=2)$, and cisternal magnesium versus no magnesium $(\mathrm{n}=2)$.

Therapies were analyzed for efficacy in reducing vasospasm (Fig. 2), DCI (Fig. 3), and cerebral infarction (Fig. 4) as well as improving outcome (Fig. 5). Intraventricular nimodipine was shown to decrease vasospasm (OR 0.59, $95 \%$ CI $0.37-0.94 ; p=0.03)$. Therapies that significantly reduced DCI were CSF drainage via LD (OR $0.47,95 \%$ CI $0.25-0.88 ; \mathrm{p}=0.02$ ) and cisternal magnesium (OR 0.27, $95 \%$ CI $0.07-1.02 ; \mathrm{p}=0.05)$. CSF drainage via LD was also found to significantly reduce the incidence of cerebral infarction (OR 0.35, 95\% 0.24-0.51; p < 0.001). Lastly, functional outcome was also significantly better in patients who received CSF drainage via LD (OR 2.42, 95\% CI 1.39-4.21; $\mathrm{p}=0.002$ ).

\section{Discussion}

Intrathecal treatment is a promising therapeutic modality in vasospasm. The use of EVDs and LDs is twofold-they allow for both CSF drainage and infusion of pharmaceutical agents. Targeted administration of agents

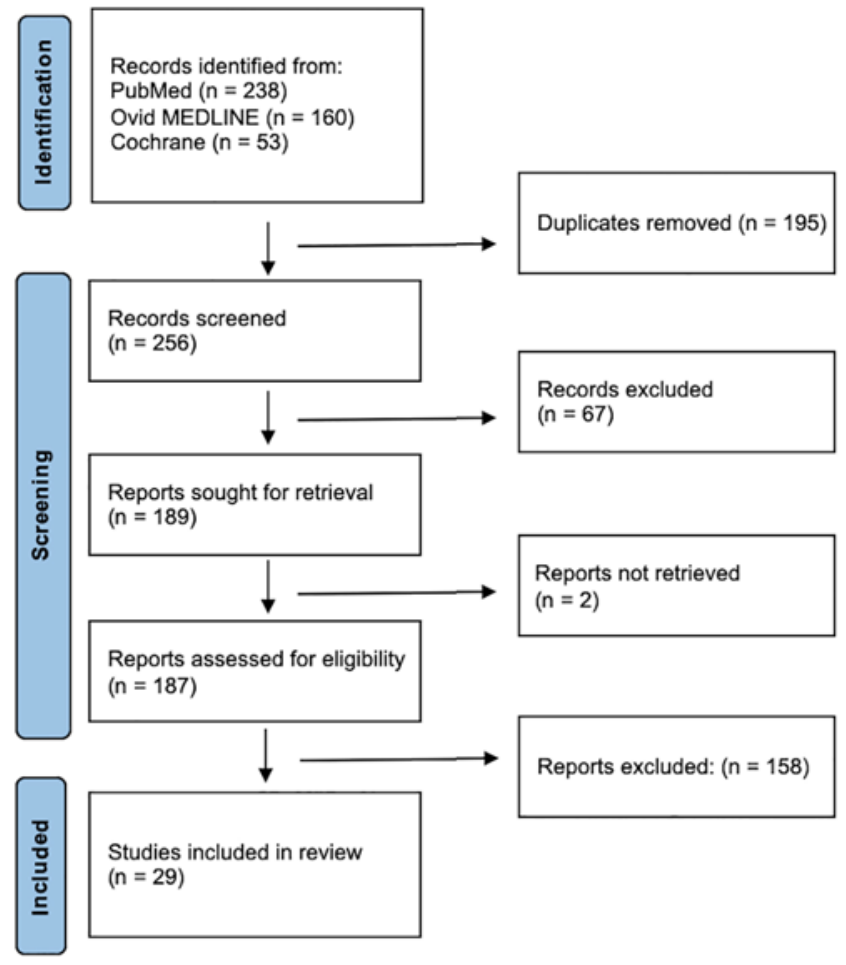

FIG. 1. Identification of studies via PRISMA guidelines. Data added to the PRISMA template [from Page MJ, McKenzie JE, Bossuyt PM, Boutron I, Hoffmann TC, Mulrow CD, et al. The PRISMA 2020 statement: an updated guideline for reporting systematic reviews. BMJ. 2021;372:n71. doi: 10.1136/bmj.n71] under the terms of the Creative Commons Attribution License.

directly into the thecal compartment negates the need for blood-brain barrier penetrance, further expanding therapeutic options. EVDs are frequently placed as part of many standard hospital protocols for ICP monitoring, and medication can be easily administered via this route. Promising intrathecal management options are highlighted below, categorized by their mechanism of action.

\section{Blood Clot Clearance}

An increased amount of hemorrhage has been reported to be the greatest predictor of vasospasm. ${ }^{3}$ Research has identified certain blood components as contributors to a proinflammatory environment in aSAH; these include acute platelet activation and release of arginase-1, which leads to dysregulated metabolism of nitric oxide precursors. ${ }^{36,37}$

\section{CSF Drainage}

Drainage via LD or EVD allows expedited removal of blood products from the CSF. In addition to blood, increased protein, sphingosine 1-phosphate, and IL-6 levels in the CSF have been associated with poor outcomes. ${ }^{38-40}$ One meta-analysis found that, when compared with no CSF drainage, patients who received CSF drainage had a decreased incidence of vasospasm and DCI with better long-term outcomes. ${ }^{41}$ Aydin et al. ${ }^{10}$ demonstrated that patients who received drainage 
TABLE 1. Prophylactic CSF drainage

\begin{tabular}{|c|c|c|c|c|c|c|c|c|c|c|}
\hline $\begin{array}{l}\text { Authors } \\
\& \text { Year }\end{array}$ & $\begin{array}{l}\text { Study } \\
\text { Design }\end{array}$ & $\begin{array}{l}\text { Aneurysm } \\
\text { Treatment }\end{array}$ & Treatment Regimen & $\begin{array}{l}\text { Experimental } \\
\text { Group }(n)\end{array}$ & Control (n) & Vasospasm & $\mathrm{DCl}$ & $\begin{array}{l}\text { Cerebral } \\
\text { Infarction }\end{array}$ & Complications & $\begin{array}{c}\text { Functional } \\
\text { Outcome }\end{array}$ \\
\hline $\begin{array}{l}\text { Amato } \\
\text { et al., } \\
2011^{7}\end{array}$ & $\begin{array}{l}\text { Observa- } \\
\text { tional }\end{array}$ & Mixed & $\begin{array}{l}\text { No standardized regimen; } \\
\text { provider dependent }\end{array}$ & $\begin{array}{l}\text { Open EVD } \\
(24)\end{array}$ & $\begin{array}{l}\text { Intermittent } \\
\text { EVD } \\
\text { drainage } \\
\text { (13) }\end{array}$ & \pm & \pm & \pm & +++ & \pm \\
\hline $\begin{array}{l}\text { Maeda } \\
\text { et al., } \\
2013^{8}\end{array}$ & $\begin{array}{l}\text { Observa- } \\
\text { tional }\end{array}$ & Coiling & $\begin{array}{l}\text { CSF drain placed day of op } \\
\text { through day } 14 \text {; target } \\
\text { drainage rate was } 120-240 \\
\text { ml/day }\end{array}$ & LD (34) & EVD (17) & \pm & \pm & \pm & \pm & +++ \\
\hline $\begin{array}{l}\text { Ormond } \\
\text { et al., } \\
2013^{9}\end{array}$ & $\begin{array}{l}\text { Retro- } \\
\text { spec- } \\
\text { tive }\end{array}$ & NR & $\begin{array}{l}\text { After failed EVD removal, LD } \\
\text { placed for a mean of } 6.7 \\
\text { days (range } 4-16 \text { days) w/ } \\
\text { a drainage rate of } 10 \mathrm{ml} / \mathrm{hr}\end{array}$ & LD (25) & NA & + & + & NR & $\begin{array}{l}\text { Decreased } \\
\text { VPS }\end{array}$ & + \\
\hline $\begin{array}{l}\text { Park } \\
\text { et al., } \\
2015^{11}\end{array}$ & RCT & Mixed & $\begin{array}{l}\text { LD placed during aneurysm } \\
\text { treatment \& left in place for } \\
14 \text { days } \mathrm{w} / \text { a drainage rate } \\
\text { of } 5-10 \mathrm{ml} / \mathrm{hr}\end{array}$ & LD (126) & $\begin{array}{l}\text { No LD } \\
\quad(108)\end{array}$ & NR & +++ & +++ & $\begin{array}{l}\text { Decreased } \\
\text { angioplasty }\end{array}$ & +++ \\
\hline $\begin{array}{l}\text { Meguro } \\
\text { et al., } \\
2016^{12}\end{array}$ & $\begin{array}{l}\text { Case } \\
\text { series }\end{array}$ & Coiling & $\begin{array}{l}\text { Mechanically ventilated pts } \\
\text { w/ neurogenic pulmonary } \\
\text { edema had LD placed } \\
\text { following coiling \& kept in } \\
\text { place for } 72 \mathrm{hrs}\end{array}$ & LD (12) & NA & NR & $N R$ & NR & Pneumonia & + \\
\hline $\begin{array}{l}\text { Borkar } \\
\text { et al., } \\
2018^{13}\end{array}$ & RCT & Clipping & $\begin{array}{l}\text { LD placed before op; before } \\
\text { dural opening, the LD was } \\
\text { opened \& kept in place for } \\
72 \text { hrs }\end{array}$ & LD (30) & No LD (30) & +++ & +++ & +++ & \pm & t++ \\
\hline $\begin{array}{l}\text { Fang } \\
\text { et al., } \\
2020^{15}\end{array}$ & $\begin{array}{l}\text { Retro- } \\
\text { spec- } \\
\text { tive }\end{array}$ & Clipping & $\begin{array}{l}\text { LD placed before op; drainage } \\
\text { rate of } 5-10 \mathrm{ml} / \mathrm{hr} \text { or until } \\
\text { fluid was not visibly hemor- } \\
\text { rhagic (LD was not kept in } \\
\text { place }>10 \text { days) }\end{array}$ & LD (113) & No LD (80) & NR & +++ & +++ & $\begin{array}{l}\text { Decreased } \\
\text { hydro- } \\
\text { cephalus }\end{array}$ & +++ \\
\hline $\begin{array}{l}\text { Jeong } \\
\text { et al., } \\
2020^{16}\end{array}$ & $\begin{array}{l}\text { Retro- } \\
\text { spec- } \\
\text { tive }\end{array}$ & Mixed & $\begin{array}{l}\text { LD placed before posthemor- } \\
\text { rhage day } 4 \text { \& continued for } \\
\geq 3 \text { days; drainage rate of } \\
5-10 \mathrm{ml} / \mathrm{hr} \text { or until fluid was } \\
\text { not visibly hemorrhagic }\end{array}$ & LD (28) & No LD (79) & NR & \pm & \pm & None & \pm \\
\hline
\end{tabular}

$\mathrm{LP}=$ lumbar puncture; $\max =$ maximum; NA = not applicable; $\mathrm{NR}=$ not reported; pts = patients; $\mathrm{RCT}=$ randomized controlled trial; VPS = ventriculoperitoneal shunt; $\pm=$ no significant difference; + = reported improvement; +++ = significant improvement $(p<0.05)$.

via LD had an overall decreased incidence of hydrocephalus and lower ICP values. Those who had visibly clear blood on CSF drainage were less likely to develop DCI. Even more importantly, those patients who did have hemorrhagic CSF were more likely to have DCI resolution once clear CSF was found. Our meta-analysis showed that drainage via LD significantly reduced DCI and cerebral infarction with a higher rate of favorable outcomes.

A computational model by Abolfazli et al. demonstrated LDs to be an effective means to facilitate blood clearance, with higher drainage rates correlated with increased clot clearance..$^{42}$ It is important to note that CSF drainage in the absence of adequate subarachnoid clot removal 
TABLE 2. Prophylactic fibrinolysis

\begin{tabular}{|c|c|c|c|c|c|c|c|c|c|c|}
\hline $\begin{array}{l}\text { Authors } \\
\& \text { Year }\end{array}$ & $\begin{array}{c}\text { Study } \\
\text { Design }\end{array}$ & $\begin{array}{l}\text { Aneurysm } \\
\text { Repair }\end{array}$ & Treatment Regimen & $\begin{array}{c}\text { Experimental } \\
\text { Group (n) }\end{array}$ & $\begin{array}{l}\text { Control } \\
\text { (n) }\end{array}$ & Vasospasm & $\mathrm{DCl}$ & $\begin{array}{l}\text { Cerebral } \\
\text { Infarction }\end{array}$ & Complications & $\begin{array}{c}\text { Functional } \\
\text { Outcome }\end{array}$ \\
\hline $\begin{array}{l}\text { Rama- } \\
\text { krishna et } \\
\text { al., } 2010^{17}\end{array}$ & $\begin{array}{l}\text { Retro- } \\
\text { spec- } \\
\text { tive }\end{array}$ & Mixed & $\begin{array}{l}\text { tPA (5 mg in } 2 \text { ml NS) } \\
\text { administered via EVD; } \\
\text { EVD flushed with } 3 \mathrm{ml} \\
\text { NS \& then both LD \& } \\
\text { EVD clamped; LD un- } \\
\text { clamped after } 30 \text { mins \& } \\
\text { EVD unclamped after } 60 \\
\text { mins; tPA regimen was } \\
\text { repeated } 24 \text { hrs apart } \times 7\end{array}$ & $\begin{array}{l}\text { tPA via EVD \& } \\
\quad \text { LD (41) }\end{array}$ & EVD (35) & +++ & NR & NR & $\begin{array}{l}\text { Decreased } \\
\text { VPS \& an- } \\
\text { gioplasty }\end{array}$ & \pm \\
\hline $\begin{array}{l}\text { Kai et al., } \\
2011^{18}\end{array}$ & RCT & Coiling & $\begin{array}{l}60,000 \text { U urokinase in } 20 \\
\text { ml NS injected into the } \\
\text { cisterna magna via mi- } \\
\text { crocatheter; continuous } \\
\text { infusion for 20-30 mins; } \\
\text { microcatheter clamped } \\
\text { for } 2 \text { hrs \& repeated until } \\
\text { 24-hr scan showed clot } \\
\text { resolution }\end{array}$ & Urokinase (6) & NA & NR & + & NR & NR & + \\
\hline $\begin{array}{l}\text { Etminan } \\
\text { et al., } \\
2013^{19}\end{array}$ & RCT & Mixed & $\begin{array}{l}5 \text { mg tPA in } 2 \text { ml NS via } \\
\text { EVD every } 12 \text { hrs via } \\
\text { EVD; EVD clamped for } \\
30 \text { mins; repeated for } \\
48 \text { hrs }\end{array}$ & $\begin{array}{l}\text { tPA via EVD } \\
\text { w/ lat rota- } \\
\text { tion therapy } \\
(30)\end{array}$ & EVD (30) & \pm & \pm & \pm & \pm & \pm \\
\hline $\begin{array}{l}\text { Litrico et } \\
\text { al., } 2013^{20}\end{array}$ & RCT & Mixed & $\begin{array}{l}3 \text { mg tPA (1 mg/ml) via EVD } \\
6-12 \text { hrs after aneurysm } \\
\text { repair; } 2 \text { ml NS flush; } \\
\text { EVD clamped for } 1 \mathrm{hr} ; \\
\text { continued every } 12 \mathrm{hrs} \\
\text { until postregimen scan } \\
\text { showed clot resolution } \\
\text { (max tPA } 36 \mathrm{mg} \text { ) }\end{array}$ & $\begin{array}{l}\text { tPA via EVD } \\
\quad(11)\end{array}$ & EVD (8) & NR & \pm & NR & \pm & \pm \\
\hline $\begin{array}{l}\text { Kramer et } \\
\text { al., } 2014^{21}\end{array}$ & RCT & Coiling & $\begin{array}{l}2 \mathrm{mg} \text { IV tissue tPA every } \\
12 \mathrm{hrs}(\mathrm{max} 10 \mathrm{mg} \text { ) or } \\
\text { placebo }\end{array}$ & $\begin{array}{l}\text { tPA via EVD } \\
\quad(6)\end{array}$ & $\begin{array}{l}\text { Placebo } \\
\text { (6) }\end{array}$ & \pm & \pm & \pm & \pm & \pm \\
\hline $\begin{array}{l}\text { Yoshikane } \\
\text { et al., } \\
2021^{22}\end{array}$ & $\begin{array}{l}\text { Retro- } \\
\text { spec- } \\
\text { tive }\end{array}$ & Clipping & $\begin{array}{l}\text { Irrigation of urokinase } \\
(120,000 \mathrm{U} / 1000 \mathrm{ml}) \text { in } \\
0.9 \% \mathrm{NS}>30 \mathrm{mins} \text { after } \\
\text { clipping }\end{array}$ & $\begin{array}{l}\text { Urokinase } \\
\quad(21)\end{array}$ & $\begin{array}{l}\text { No uroki- } \\
\text { nase } \\
\text { (19) }\end{array}$ & \pm & \pm & +++ & $\begin{array}{l}\text { Decreased } \\
\text { pneumonia } \\
\text { \& HF }\end{array}$ & +++ \\
\hline
\end{tabular}

$\mathrm{HF}=$ heart failure; IV = intraventricular; NS = normal saline.

can lead to an increased CSF pH and lower CSF $\mathrm{PCO}_{2} \cdot{ }^{43}$ Therefore, if CSF drainage is not sufficiently removing blood, it has a paradoxically increased risk of acid metabolite buildup, arterial constriction, and DCI. ${ }^{43}$

\section{Fibrinolysis}

Fibrinolysis accelerates the rate of clot clearance though enzymatic breakdown. This was seen in 3 studies included in our review. ${ }^{19-21}$ These medications also inhibit acute platelet activation and the inflammatory cascade. Increased intrathecal levels of cytokines following aSAH and fever have been found to be an independent risk factor for DCI.4 Low-frequency head rotation has also been tested in conjunction with intraventricular tPA. This ki- netic mechanism is thought to augment the effect of fibrinolytics and has been shown to increase the rate of clot clearance. ${ }^{19,45}$ Eicker et al. ${ }^{45}$ showed promising results for DCI in an interim report for a randomized controlled trial involving tPA and lateral rotational head therapy; however, the final results showed no difference in vasospasm, cerebral infarction, or functional outcome. ${ }^{46}$

Our review showed that irrigation with urokinase $(120,000 \mathrm{U} / 1000 \mathrm{ml})$ in $0.9 \%$ normal saline for more than 30 minutes after clipping significantly decreased DCI and improved clinical outcome. Ramakrishna et al. ${ }^{17}$ demonstrated decreased severe vasospasm on TCD ultrasonography by administering tPA (5 $\mathrm{mg}$ in $2 \mathrm{ml}$ normal saline) via EVD. Currently, Gaberel et al. ${ }^{47}$ are 
TABLE 3. Calcium channel antagonists

\begin{tabular}{|c|c|c|c|c|c|c|c|c|c|c|}
\hline $\begin{array}{l}\text { Authors } \\
\& \text { Year }\end{array}$ & $\begin{array}{l}\text { Study } \\
\text { Design }\end{array}$ & $\begin{array}{l}\text { Aneurysm } \\
\text { Treatment }\end{array}$ & $\begin{array}{l}\text { Treatment } \\
\text { Regimen }\end{array}$ & $\begin{array}{c}\text { Experimental } \\
\text { Group (n) }\end{array}$ & $\begin{array}{l}\text { Control } \\
\text { (n) }\end{array}$ & Vasospasm & $\mathrm{DCl}$ & $\begin{array}{l}\text { Cerebral } \\
\text { Infarction }\end{array}$ & Complications & $\begin{array}{c}\text { Functional } \\
\text { Outcome }\end{array}$ \\
\hline \multicolumn{11}{|l|}{$\begin{array}{c}\text { Rescue man- } \\
\text { agement }\end{array}$} \\
\hline $\begin{array}{l}\text { Webb et } \\
\text { al., } 2010^{23}\end{array}$ & $\begin{array}{l}\text { Retrospec- } \\
\text { tive }\end{array}$ & Mixed & $\begin{array}{l}4 \text { mg nicardipine mixed } \\
\text { w/ } 2 \text { NS given via } \\
\text { EVD every } 8-12 \text { hrs; } \\
\text { EVD clamped for } \\
30 \text { mins }\end{array}$ & $\begin{array}{l}\text { Nicardipine } \\
(64)\end{array}$ & NA & +++ & $N R$ & NR & $\begin{array}{l}\text { Ventriculosto- } \\
\text { my-related } \\
\text { infection }\end{array}$ & NR \\
\hline $\begin{array}{l}\text { Lu et al., } \\
2012^{24}\end{array}$ & $\begin{array}{l}\text { Retrospec- } \\
\text { tive }\end{array}$ & Mixed & $\begin{array}{l}\text { No standardized } \\
\text { protocol; median } \\
\text { nicardipine dose was } \\
4 \mathrm{mg} \text { (range } 3-7 \mathrm{mg} \text { ), } \\
\text { w/ } 7 \text { median doses } \\
\text { (range 1-17) }\end{array}$ & $\begin{array}{l}\text { Nicardipine } \\
\text { (14) }\end{array}$ & $\begin{array}{l}\text { No nicar- } \\
\text { dipine } \\
(14)\end{array}$ & +++ & NR & NR & None & \pm \\
\hline $\begin{array}{l}\text { Sadan et } \\
\text { al., } 2022^{26}\end{array}$ & $\begin{array}{l}\text { Retrospec- } \\
\text { tive }\end{array}$ & Mixed & $\begin{array}{l}4 \text { mg nicardipine in } 2 \mathrm{ml} \\
\mathrm{NaCl} 0.9 \% \text { solution } \\
\text { via EVD every } 8 \mathrm{hrs} \text {; } \\
\text { EVD flushed w/ } 1-2 \\
\mathrm{ml} \mathrm{NaCl} 0.9 \% \text {; EVD } \\
\text { clamped for } 30 \text { mins }\end{array}$ & $\begin{array}{l}\text { Nicardipine } \\
\quad(422)\end{array}$ & $\begin{array}{l}\text { No nicar- } \\
\text { dipine } \\
\text { (SAHIT } \\
\text { data- } \\
\text { base) } \\
(929)\end{array}$ & +++ & + & NR & $\begin{array}{l}\text { Increased } \\
\text { VPS; } \\
\text { decreased } \\
\text { ventriculitis }\end{array}$ & + \\
\hline \multicolumn{11}{|l|}{ Prophylaxis } \\
\hline $\begin{array}{l}\text { Barth et } \\
\text { al., } 2011^{27}\end{array}$ & $\begin{array}{l}\text { Prospec- } \\
\text { tive }\end{array}$ & Mixed & $\begin{array}{l}4 \text { mg sustained-release } \\
\text { nicardipine pellets; } \\
\text { pts received either } 6 \\
\text { or } 10 \text { pellets }\end{array}$ & $\begin{array}{l}\text { IV nicardi- } \\
\text { pine (31) }\end{array}$ & $\begin{array}{l}\text { No nicar- } \\
\text { dipine } \\
(16)\end{array}$ & $\begin{array}{l}+++ \\
\text { (clipping } \\
\text { only) }\end{array}$ & NR & NR & NR & NR \\
\hline
\end{tabular}

enrolling patients in a phase III randomized controlled trial of intraventricular tPA. The study will compare an EVD alone and an EVD in addition to tPA. This will be the first phase III trial assessing the efficacy of this treatment in the prevention of DCI and other aSAH-related complications.

\section{Targeting Heme Degradation Products}

Other intrathecal therapeutic agents targeting specific heme degradation products have been tested in vitro and in animal models. These include haptoglobin, deferoxamine, and vitamin D. An in vitro study by Garland et al. ${ }^{48}$ found that haptoglobin has the ability to bind to CSF hemopexin and could potentially improve outcomes. Studies have suggested that increased iron levels in CSF can be associated with secondary brain injury. ${ }^{49}$ The iron chelator, deferoxamine, was found to be neuroprotective in a mouse model; however, this protective effect was not directly associated with vasospasm.

\section{Vasodilators}

\section{Calcium Channel Antagonists}

Since the 1980s, nimodipine has been the mainstay for prevention of vasospasm and cerebral infarction secondary to aSAH. It is now considered the standard of care for management of patients with ruptured aneurysms. Interestingly, its mechanism of action is not thought to be due to its vasodilatory effect, and it remains incompletely understood. ${ }^{4}$ Its chemical makeup made nimodipine a favorable drug for vasospasm as it readily crosses the blood-brain barrier. With the development of more advanced catheters and delivery systems, other calcium channel antagonists have been tested in animal studies and in clinical practice, including intrathecal verapamil and nicardipine. ${ }^{24,25,50-52}$ The calcium channel antagonists in our review showed a significant vasodilatory effect and decrease in vasospasm; however, none of these translated to decreased DCI or improved clinical outcome.

Kasuya et al. ${ }^{53}$ pioneered intrathecal therapy with calci- 
TABLE 4. Other vasodilators

\begin{tabular}{|c|c|c|c|c|c|c|c|c|c|c|}
\hline $\begin{array}{l}\text { Authors } \\
\& \text { Year }\end{array}$ & $\begin{array}{l}\text { Study } \\
\text { Design }\end{array}$ & $\begin{array}{l}\text { Aneurysm } \\
\text { Treatment }\end{array}$ & $\begin{array}{l}\text { Treatment } \\
\text { Regimen }\end{array}$ & $\begin{array}{c}\text { Experimental } \\
\text { Group (n) }\end{array}$ & $\begin{array}{l}\text { Control } \\
\text { (n) }\end{array}$ & Vasospasm & $\mathrm{DCl}$ & $\begin{array}{l}\text { Cerebral } \\
\text { Infarction }\end{array}$ & Complications & $\begin{array}{c}\text { Functional } \\
\text { Outcome }\end{array}$ \\
\hline \multicolumn{11}{|l|}{$\begin{array}{l}\text { Rescue } \\
\text { manage- } \\
\text { ment }\end{array}$} \\
\hline $\begin{array}{l}\text { Sadama- } \\
\text { sa et al., } \\
2014^{30}\end{array}$ & $\begin{array}{l}\text { Feasibility/ } \\
\text { safety }\end{array}$ & Mixed & $\begin{array}{l}0.87 \text { mg milrinone } \\
\text { (2.6 ml/day) per } 7 \mathrm{ml} \\
\text { NS for } 2 \text { hrs via LD; } \\
\text { LD clamped for } 2 \text { hrs; } \\
\text { cycle repeated until } \\
\text { day } 14 \text { after SAH }\end{array}$ & Milrinone (170) & NA & NR & \pm & \pm & None & + \\
\hline $\begin{array}{l}\text { Ehlert } \\
\text { et al., } \\
2016^{31}\end{array}$ & $\begin{array}{l}\text { Case } \\
\text { report }\end{array}$ & Coiling & $\begin{array}{l}\text { Continuous intravenous } \\
\text { molsidomine \& boluses } \\
\text { of sodium nitroprus- } \\
\text { side } 7 \text { times over } 22 \\
\text { days for } \mathrm{DCl} \text { ( } 3 \text { or } 5 \mathrm{mg} \\
\text { each time) }\end{array}$ & $\begin{array}{l}\text { IV sodium nitro- } \\
\text { prusside w/ } \\
\text { intravenous } \\
\text { molsidomine } \\
\text { (1) }\end{array}$ & NA & + & + & + & NR & + \\
\hline $\begin{array}{l}\text { Ehlert } \\
\text { et al., } \\
2020^{33}\end{array}$ & $\begin{array}{l}\text { Retrospec- } \\
\text { tive, } \\
\text { obser- } \\
\text { vational }\end{array}$ & Mixed & $\begin{array}{l}\text { Continuous intravenous } \\
\text { molsidomine \& boluses } \\
\text { of sodium nitroprus- } \\
\text { side w/ DCl (2-10 mg); } \\
\text { mean } 5.6 \mathrm{mg}\end{array}$ & $\begin{array}{l}\text { IV sodium nitro- } \\
\text { prusside w/ } \\
\text { intravenous } \\
\text { molsidomine } \\
\text { (18) }\end{array}$ & NA & t++ & + & + & Septic shock & + \\
\hline \multicolumn{11}{|l|}{ Prophylaxis } \\
\hline
\end{tabular}

$\mathrm{LR}=$ lactated Ringer's.

um channel antagonists in 2002 when they first described prolonged-release nicardipine pellets as an effective means to decrease vasospasm and DCI. Further building on this work, Kuori et al. ${ }^{54}$ demonstrated that a nicardipine prolonged-release implant (NPRI) significantly decreased vasospasm and DCI in patients with aSAH undergoing surgical clipping. Additionally, these patients had improved $\mathrm{mRS}$ scores at discharge. A phase II trial is currently recruiting to further assess this treatment modality. After aneurysm clipping, 10 NPRI capsules will be placed into the basal cistern, directly abutting the blood vessel walls (registration no. NCT04269408, ClinicalTrials.gov).

Intrathecal use of both dotarizine and flunarizine has been examined in animal models. ${ }^{55,56}$ Both of these were compared with the standard of care, nimodipine. It was found that dotarizine and flunarizine have marked vasodilatory effects in an experimental model and have the potential to be valid alternatives to nimodipine..$^{55,56}$

\section{Other Vasodilators}

In addition to calcium channel antagonists, other vasodilators that are currently being studied include milrinone, ${ }^{30,32}$ nitric oxide, ${ }^{33}$ magnesium sulfate, ${ }^{57}$ cilostazol, ${ }^{58}$ and glyceryl trinitrate. ${ }^{59}$ Milrinone is a phosphodiesterase-3 inhibitor that has been proven to be safe and feasible for the prevention of DCI and in the period following confirmed vasospasm in a clinical setting. Koyanagi et al. ${ }^{32}$ showed that a treatment regimen of $0.87 \mathrm{mg}$ milrinone per 
TPA+EVD EVD alone Odds Ratio

Study or Subgroup Events Total Events Total Weight IV, Random, 95\% CI Year

$\begin{array}{llllllll}\text { Etminan, et al. }{ }^{19} & 12 & 30 & 16 & 30 & 77.0 \% & 0.58[0.21,1.62] & 2013\end{array}$

Kramer, et al. ${ }^{21}$

$\begin{array}{lllll}2 & 6 & 5 & 6 & 23.0 \%\end{array}$

$0.10[0.01,1.54] 2014$

Total $(95 \% \mathrm{Cl})$

36

$36 \quad 100.0 \%$

$0.39[0.09,1.67]$

Total events

14

21

Heterogeneity: $\mathrm{Tau}^{2}=0.44 ; \mathrm{Chi}^{2}=1.40, \mathrm{df}=1(\mathrm{P}=0.24) ; \mathrm{I}^{2}=29 \%$

Test for overall effect: $Z=1.27(P=0.20)$

Intra Nimodipine Oral Nimodipine

Odds Ratio

\begin{tabular}{|c|c|c|c|c|c|c|c|c|c|c|}
\hline Study or Subgroup & Events & Total & Events & Total & Weight & IV, Random, $95 \% \mathrm{CI}$ & Year & IV, Random & $\mathrm{m}, 95 \% \mathrm{Cl}$ & \\
\hline Carlson, et al. 28 & 69 & 138 & 91 & 144 & $98.4 \%$ & $0.58[0.36,0.94]$ & 2020 & $E$ & & \\
\hline Macdonald, et al. ${ }^{29}$ & 4 & 5 & 1 & 1 & $1.6 \%$ & $1.00[0.02,40.28]$ & 2020 & & & \\
\hline Total $(95 \% \mathrm{Cl})$ & & 143 & & 145 & $100.0 \%$ & $0.59[0.37,0.94]$ & & & & \\
\hline Total events & 73 & & 92 & & & & & & & \\
\hline \multicolumn{8}{|c|}{$\begin{array}{l}\text { Heterogeneity: } \text { Tau }^{2}=0.00 ; \mathrm{Chi}^{2}=0.08, \mathrm{df}=1(\mathrm{P}=0.78) ; \mathrm{I}^{2}=0 \text { ? } \\
\text { Test for overall effect: } \mathrm{Z}=2.21(\mathrm{P}=0.03)\end{array}$} & $\begin{array}{l}0.5 \text { i } \\
\text { No VSP }\end{array}$ & VSP $^{2}$ & 5 \\
\hline
\end{tabular}

FIG. 2. Forest plots showing pooled ORs of vasospasm (VSP) by therapeutic regimen. Intra = intraventricular; IV = inverse variance.

LD No LD Odds Ratio

Study or Subgroup Events Total Events Total Weight IV, Random, $95 \% \mathrm{Cl}$ Year

\begin{tabular}{|c|c|c|c|c|c|c|}
\hline Park, et al. ${ }^{11}$ & 24 & 126 & 45 & 108 & $31.1 \%$ & $0.33[0.18,0.59]$ \\
\hline Borkar, et al. ${ }^{13}$ & 9 & 30 & 19 & 30 & $18.8 \%$ & $0.25[0.08,0.73]$ \\
\hline Fang, et al. ${ }^{15}$ & 48 & 113 & 36 & 80 & $31.4 \%$ & $0.90[0.51,1.61]$ \\
\hline Jeong, et al. ${ }^{16}$ & 5 & 28 & 22 & 79 & $18.7 \%$ & $0.56[0.19,1.67]$ \\
\hline Total $(95 \% \mathrm{Cl})$ & & 297 & & 297 & $100.0 \%$ & $0.47[0.25,0.88]$ \\
\hline $\begin{array}{l}\text { Total events } \\
\text { Heterogeneity: } \\
\text { Test for overall }\end{array}$ & 86 & & 122 & & & $1 \%$ \\
\hline
\end{tabular}

Test for overall effect: $Z=2.35(P=0.02)$

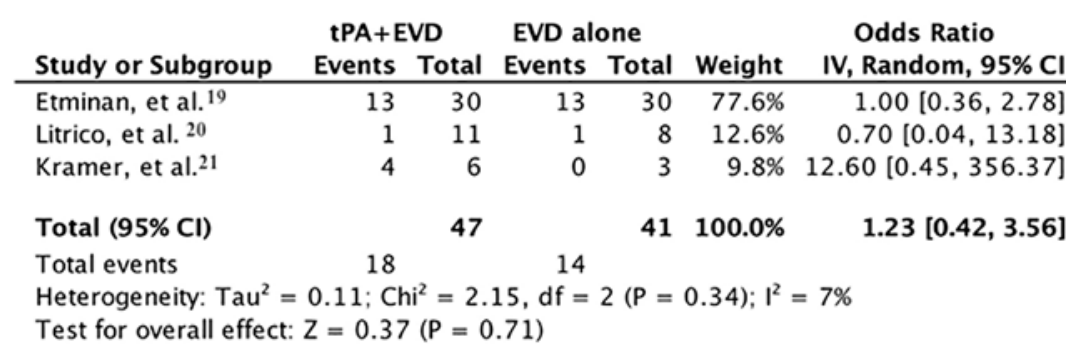

Intra Nimodipine Oral Nimodipine

Odds Ratio

Study or Subgroup Events Total Events Total Weight IV, Random, 95\% CI Year

$\begin{array}{lllrrrrr}\text { Carlson, et al. }{ }^{28} & 32 & 138 & 40 & 144 & 97.9 \% & 0.78[0.46,1.34] & 2020\end{array}$

Macdonald, et al. 29

Total $(95 \% \mathrm{Cl})$

143

Total events

40

$1 \quad 2.1 \% \quad 1.00[0.02,40.28] 2020$

$145 \quad 100.0 \%$

$0.79[0.46,1.34]$

Heterogeneity: $\mathrm{Tau}^{2}=0.00 ; \mathrm{Chi}^{2}=0.02, \mathrm{df}=1(\mathrm{P}=0.90) ; \mathrm{I}^{2}=0 \%$

Test for overall effect: $Z=0.87(P=0.38)$

\section{Cisternal Mg No Mg}

Odds Ratio

Study or Subgroup Events Total Events Total Weight IV, Random, 95\% CI Year

\begin{tabular}{|c|c|c|c|c|c|c|c|}
\hline study or subgroup & Events & tal & Events & & Weight & IV, Random, 95\% Cl & Ye \\
\hline Yamamoto, et al. ${ }^{34}$ & 5 & 35 & 9 & 35 & $58.2 \%$ & $0.48[0.14,1.62]$ & 2016 \\
\hline Takeuchi, et al. ${ }^{35}$ & 3 & 24 & 7 & 13 & $41.8 \%$ & $0.12[0.02,0.62]$ & 2021 \\
\hline Total $(95 \% \mathrm{CI})$ & & 59 & & 48 & $100.0 \%$ & $0.27[0.07,1.02]$ & \\
\hline $\begin{array}{l}\text { Total events } \\
\text { Heterogeneity: Tau² } \\
\text { Test for overall effect }\end{array}$ & $\begin{array}{r}8 \\
0.40 ; \mathrm{Ch} \\
Z=1.93\end{array}$ & $\begin{array}{l}{ }^{2}=1.7 \\
(P=0.6\end{array}$ & $\begin{array}{l}16 \\
5, \mathrm{df}=1 \\
05)\end{array}$ & $(\mathrm{P}=$ & (9); $1^{2}=$ & $43 \%$ & \\
\hline
\end{tabular}

Test for overall effect: $Z=1.93(P=0.05)$

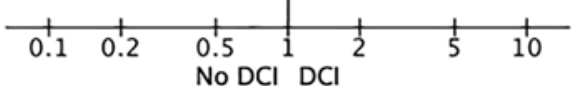

FIG. 3. Forest plots showing pooled ORs of $\mathrm{DCl}$ by therapeutic regimen. 


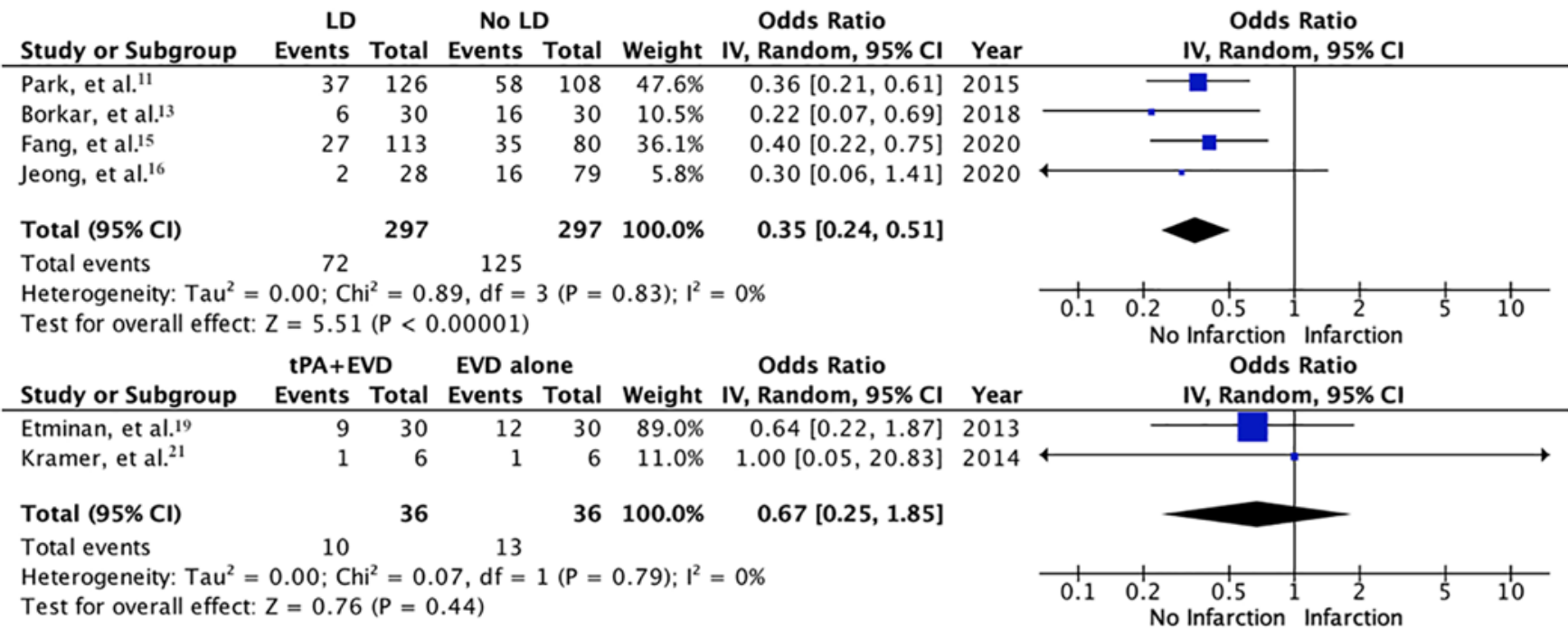

FIG. 4. Forest plots showing pooled ORs of cerebral infarction by therapeutic regimen.

\begin{tabular}{|c|c|c|c|c|c|c|c|c|c|c|}
\hline Study or Subgroup & $\begin{array}{l}\text { LD } \\
\text { Events }\end{array}$ & Total & $\begin{array}{l}\text { No LD } \\
\text { Events }\end{array}$ & Total & Weight & $\begin{array}{l}\text { Odds Ratio } \\
\text { IV, Random, } 95 \% \mathrm{CI}\end{array}$ & Year & $\begin{array}{r}\text { Odds } \\
\text { IV, Randon }\end{array}$ & $\begin{array}{l}\text { Ratio } \\
\mathrm{m}, 95 \% \mathrm{Cl}\end{array}$ & \\
\hline Park, et al. ${ }^{11}$ & 116 & 126 & 91 & 108 & $44.9 \%$ & $2.17[0.95,4.96]$ & 2015 & & 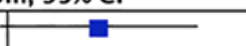 & \\
\hline Fang, et al. .15 & 105 & 113 & 63 & 80 & $38.3 \%$ & $3.54[1.44,8.68]$ & 2020 & & 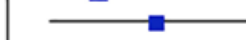 & \\
\hline Jeong, et al. ${ }^{16}$ & 25 & 28 & 68 & 79 & $16.7 \%$ & $1.35[0.35,5.23]$ & 2020 & & & \\
\hline Total $(95 \% \mathrm{Cl})$ & & 267 & & 267 & $100.0 \%$ & $2.42[1.39,4.21]$ & & & & \\
\hline Total events & 246 & & 222 & & & & & & & \\
\hline $\begin{array}{l}\text { Heterogeneity: } \mathrm{Tau}^{2}= \\
\text { Test for overall effect }\end{array}$ & $\begin{array}{l}0.00 ; C h \\
Z=3.12\end{array}$ & $\begin{array}{l}\mathrm{i}^{2}=1.4 \\
(P=0 .\end{array}$ & $\begin{array}{l}48, \mathrm{df}=2 \\
.002)\end{array}$ & $2(P=$ & $0.48) ; 1^{2}=$ & $=0 \%$ & & $\begin{array}{ccc}0.1 & 0.2 & 0.5 \\
& \text { Unfavorable } & \text { Outcome }\end{array}$ & $\begin{array}{ccc}1 & 2 & 5 \\
\text { Favorable Outcome }\end{array}$ & 10 \\
\hline Study or Subgroup & $\begin{array}{l}\text { TPA+E } \\
\text { Events }\end{array}$ & $\begin{array}{l}\text { VD } \\
\text { Total }\end{array}$ & $\begin{array}{l}\text { EVD alo } \\
\text { Events }\end{array}$ & $\begin{array}{l}\text { one } \\
\text { Total }\end{array}$ & Weight & $\begin{array}{c}\text { Odds Ratio } \\
\text { IV, Random, } 95 \% \mathrm{Cl}\end{array}$ & Year & $\begin{array}{r}\text { Odds } \\
\text { IV, Random }\end{array}$ & $\begin{array}{l}\text { Ratio } \\
\mathrm{m}, 95 \% \mathrm{Cl}\end{array}$ & \\
\hline Etminan, et al. ${ }^{19}$ & 18 & 28 & 14 & 26 & $65.5 \%$ & $1.54[0.52,4.60]$ & 2013 & & & \\
\hline Litrico, et al. ${ }^{20}$ & 4 & 11 & 2 & 8 & $19.2 \%$ & $1.71[0.23,12.89]$ & 2013 & & & \\
\hline Kramer, et al. ${ }^{21}$ & 3 & 6 & 3 & 6 & $15.3 \%$ & $1.00[0.10,9.61]$ & 2014 & & & \\
\hline Total $(95 \% \mathrm{Cl})$ & & 45 & & 40 & $100.0 \%$ & $1.47[0.61,3.57]$ & & & & \\
\hline Total events & 25 & & 19 & & & & & & & \\
\hline $\begin{array}{l}\text { Heterogeneity: } \mathrm{Tau}^{2}= \\
\text { Test for overall effect }\end{array}$ & $\begin{array}{l}0.00 ; C h \\
Z=0.86\end{array}$ & $\begin{array}{l}i^{2}=0.1 \\
(P=0 .\end{array}$ & $\begin{array}{l}14, \mathrm{df}=2 \\
.39)\end{array}$ & $2(P=$ & $0.93) ; 1^{2}=$ & $=0 \%$ & & $\begin{array}{ccc} & 1 & 1 \\
0.1 & 0.2 & 0.5 \\
& \text { Unfavorable } & \text { Outcome }\end{array}$ & $\begin{array}{ccc}1 & 1 & 1 \\
& 2 & 5 \\
\text { Favorable Outcome }\end{array}$ & 10 \\
\hline Study or Subgroup & $\begin{array}{l}\text { tra Nimod } \\
\text { Events }\end{array}$ & $\begin{array}{l}\text { ipine } \\
\text { Total }\end{array}$ & $\begin{array}{r}\text { Oral Nim } \\
\text { Events }\end{array}$ & $\begin{array}{l}\text { nodipir } \\
5\end{array}$ & $\begin{array}{l}\text { ine } \\
\text { lotal Weig }\end{array}$ & $\begin{array}{c}\text { Odds Ratio } \\
\text { ight IV, Random, } 95 \% \mathrm{C}\end{array}$ & $\mathrm{Cl}$ Year & $\begin{array}{r}\text { Odds } \\
\text { IV, Rando }\end{array}$ & $\begin{array}{l}\text { ds Ratio } \\
\text { lom, } 95 \% \mathrm{Cl}\end{array}$ & \\
\hline Carlson, et al. ${ }^{28}$ & 65 & 144 & 62 & & 14583. & $.3 \% \quad 1.10[0.69,1.75$ & 75] 2020 & & & \\
\hline Macdonald, et al. ${ }^{29}$ & 1 & 5 & 1 & & 116. & $0.11[0.00,4.48$ & 48] 2020 & & & \\
\hline Total $(95 \% \mathrm{Cl})$ & & 149 & & & 146100. & $0.75[0.14,4.02$ & & & & \\
\hline Total events & 66 & & 63 & & & & & & & \\
\hline $\begin{array}{l}\text { Heterogeneity: } \mathrm{Tau}^{2}= \\
\text { Test for overall effect: }\end{array}$ & $\begin{array}{l}0.83 ; \mathrm{Chi}^{2} \\
\mathrm{Z}=0.34\end{array}$ & $\begin{array}{l}=1.46 \\
P=0.7\end{array}$ & $\begin{array}{l}\text {, } d f=1(f)\end{array}$ & $P=0.2$ & 23): $1^{2}=31$ & $31 \%$ & & $\begin{array}{ccc}1 & 1 & 1 \\
0.1 & 0.2 & 0.5 \\
& \text { Unfavorable } & \text { Outcome }\end{array}$ & $\begin{array}{ccc}1 & 1 & 1 \\
1 & 2 & 5 \\
e\end{array}$ & 10 \\
\hline Study or Subgroup & $\begin{array}{l}\text { Cisterna } \\
\text { Events }\end{array}$ & $\begin{array}{l}\text { Mg } \\
\text { Total }\end{array}$ & $\begin{array}{c}\text { No M } \\
\text { Events }\end{array}$ & Total & Weight & $\begin{array}{c}\text { Odds Ratio } \\
\text { IV, Random, } 95 \% \mathrm{CI}\end{array}$ & Year & $\begin{array}{r}\text { Odds } \\
\text { IV, Randon }\end{array}$ & $\begin{array}{l}\text { Ratio } \\
\mathrm{m}, 95 \% \mathrm{CI}\end{array}$ & \\
\hline Yamamoto, et al. ${ }^{34}$ & 27 & 35 & 28 & 35 & $52.9 \%$ & $0.84[0.27,2.65]$ & 2016 & $\square$ & & \\
\hline Takeuchi, et al. ${ }^{35}$ & 17 & 24 & 3 & 13 & $47.1 \%$ & $8.10[1.70,38.60]$ & 2021 & & & \\
\hline Total $(95 \% \mathrm{Cl})$ & & 59 & & 48 & $100.0 \%$ & $2.45[0.27,22.37]$ & & & & \\
\hline Total events & 44 & & 31 & & & & & & & \\
\hline \multicolumn{8}{|c|}{$\begin{array}{l}\text { Heterogeneity: } \text { Tau }^{2}=2.07 ; \mathrm{Chi}^{2}=5.24, \mathrm{df}=1(\mathrm{P}=0.02) ; \mathrm{I}^{2}=81 \% \\
\text { Test for overall effect: } Z=0.79(\mathrm{P}=0.43)\end{array}$} & $\begin{array}{ccc}1 & 1 & 1 \\
0.1 & 0.2 & 0.5 \\
& \text { Unfavorable } & \text { Outcome }\end{array}$ & $\begin{array}{ccc}1 & 1 & 1 \\
\text { Favorable Outcome }\end{array}$ & 10 \\
\hline
\end{tabular}

FIG. 5. Forest plots showing pooled ORs of favorable outcome by therapeutic regimen. 
$7 \mathrm{ml}$ normal saline for 2 hours via LD was an effective way to decrease incidence of DCI and cerebral infarction. Nitric oxide and magnesium sulfate have also been shown to be effective in patients with aSAH. Cilostazol and glyceryl trinitrate have only been tested in animal models.

\section{Proper Catheter Administration and Maintenance}

The optimal management of LDs and EVDs remains controversial. The variables described below have been studied and should be considered during treatment.

\section{Patient Positioning}

Tangen et al. ${ }^{60}$ suggested the importance of patient positioning in a study in which they created a CSF dynamics model to assess differing situations of contaminant clearance with lumbar drainage in addition to the rate of blood clearance. The authors found that body position and drainage rates were both essential for effective blood clearance. Their findings emphasized having the patient in an upright position to mitigate infection as any contaminant present is maximally diverted from the cranial CSF compartment. ${ }^{60}$

\section{Intracranial Pressure}

Elevated ICP is a common occurrence following aSAH and can contribute to poor outcomes in the acute and subacute settings. In traumatic brain injury, increased ICP is defined as pressure $>20 \mathrm{~mm} \mathrm{Hg}$. Some estimates suggest that more than half of all patients with aSAH will experience an ICP value $>20 \mathrm{~mm} \mathrm{Hg}$ at some point during their hospital stay ${ }^{61}$ During intrathecal therapy, an EVD typically must be clamped for some time to allow the medication to dwell. For this reason, intrathecal management is typically precluded in this patient population. Additionally, vasodilators - a commonly used class of intrathecal medications - can increase ICP. Ko et al. saw an increase in ICP for approximately 1 hour following injection of intraventricular nicardipine. ${ }^{25}$ However, this effect was transient and did not significantly alter cerebral blood flow or oxidative glucose metabolism, nor did it cause systemic hypotension. Other interventions to counteract increased ICP are mitigating the dwell time by using two catheters or with concomitant use of EVDs and LDs. ${ }^{27-29}$

\section{Pressure Gradient}

A lumbar drainage catheter is sometimes placed intraoperatively to decrease fluid pressure on the brain in patients with aSAH. In rare cases, catheter placement can lead to intracranial hypotension, resulting in brainstem herniation ("brain sag"), causing neurological injury and even death. Cases of transient herniation have been reported due to lumbar catheter overdrainage. ${ }^{62}$ Leaving the CSF bag at the level of the head and using careful pressuregradient monitoring can help avoid poor outcomes. In a safety and feasibility study of lumbar drainage in patients with poor-grade aSAH, the ideal ventricolumbar pressure gradient was found to be $6 \mathrm{~mm} \mathrm{Hg}$ or less. ${ }^{63}$

\section{Infection}

Infections and medical complications following aSAH are common. The infection risk associated with intrathecal therapy is likely due to accessing the EVD or LD multiple times throughout treatment to deliver medication. Newer drains, which have the dual capability of draining and infusing, may mitigate this risk. Prolonged LD placement has also been associated with increased infection rate. This provides rationale for why Liang et al. found a decreased infection rate following serial lumbar puncture when compared with LD. ${ }^{14}$ Park et al. ${ }^{11}$ described complications they encountered during lumbar drainage. These included CSF infection and microorganism growth when they conducted a routine drainage tip culture. However, CSF infection was easily managed with proper antibiotics, and microorganism growth resolved on its own. ${ }^{11}$ Moreover, antibioticcoated EVDs are routine at many institutions. ${ }^{17}$

\section{Future of Vasospasm Management}

The future role of intrathecal management in aSAH and vasospasm is yet to be fully understood. There is a large amount of heterogeneity in the current studies exploring the topic, sometimes making results difficult to interpret; this includes medication timing and dosage in addition to catheter use. We recognize broad potential for protocolized implementation of these treatments in future trials.

There is no definitive evidence as to whether CSF drainage or medication administration via EVD or LD leads to better outcomes. LDs and EVDs have both shown safety and efficacy in intrathecal management. However, there is still controversy over the preferred method for clearing subarachnoid blood and preventing vasospasm. There have been few studies evaluating them side by side. When Maeda et al. ${ }^{8}$ retrospectively examined this, they found no significant difference in the occurrence of vasospasm, hydrocephalus, or ventriculoperitoneal shunt placement. However, the LD group had faster clot clearance and improved outcomes compared with the EVD group. It was hypothesized that the EVD could lead to hemorrhage stasis. There is currently a randomized controlled trial in the recruitment phase that will examine whether LD or EVD is superior in vasospasm prevention and clinical outcome (registration no. NCT03065231, ClinicalTrials.gov). Concomitant use of LDs and EVDs has also been shown to be effective in management of vasospasm and is an expected area of study.

Advances in biomedical engineering have led to the invention of new catheters. Stereotactic catheter ventriculocisternostomy is a method of obtaining access to the intrathecal space that was recently found to be feasible in a majority of patients with aSAH ${ }^{64}$ Roelz et al. have employed this method with nimodipine and CSF drainage with success. ${ }^{65,66}$

There are a number of other intrathecal therapies that have been explored in vitro and in animal models but have yet to be tested in patients; these include MEK 1/2 inhibitors, ${ }^{67,68}$ NDP-MSH,${ }^{69}$ ebselen,${ }^{70}$ nicergoline, ${ }^{71}$ acetylcholine, ${ }^{72}$ osteopontin, ${ }^{73}$ and valproic acid. ${ }^{74}$ In addition to these, there are experimental drugs currently being tested in an intravenous form that have yet to be tested intrathecally, including endothelin antagonists and statin therapy.

\section{Study Limitations}

There is an inherent limitation to systematic reviews 
and meta-analyses as they are retrospective. There is the potential to miss relevant studies due to the choice of search terms and an inability to access certain articles or journals. The biggest limitation encountered in this report was the heterogeneity of study designs. This was true not only for therapeutic regimens but also for how clinic outcomes were reported. Studies also continue to use different definitions for vasospasm, DCI, and cerebral infarction, which can make interpretation difficult.

\section{Conclusions}

This study provides a review of the current options for intrathecal management of aSAH-induced vasospasm. The intrathecal route has obvious advantages in that medications can be delivered directly to the subarachnoid space and do not need to cross the blood-brain barrier. Thus far, the literature is quite heterogeneous with regard to dosing, timing, and delivery method, and no single protocol emerges as the superior therapy. One therapy that has been shown to decrease DCI and cerebral infarction with improved functional outcome is CSF drainage via LD. Other therapies show promise for decreasing the incidence of vasospasm, DCI, and cerebral infarction, although there is not yet significant evidence to recommend their use in routine practice. More randomized controlled trials are warranted to determine the best combination of pharmaceutical agents and administration routes in order to formulate a standardized treatment approach.

\section{References}

1. Ziu E, Mesfin FB. Subarachnoid Hemorrhage. In: StatPearls. StatPearls Publishing; 2021. Accessed October 26, 2021. http: //www.ncbi.nlm.nih.gov/books/NBK441958/

2. Vergouwen MDI, Vermeulen M, van Gijn J, et al. Definition of delayed cerebral ischemia after aneurysmal subarachnoid hemorrhage as an outcome event in clinical trials and observational studies: proposal of a multidisciplinary research group. Stroke. 2010;41(10):2391-2395.

3. Findlay JM, Nisar J, Darsaut T. Cerebral vasospasm: a review. Can J Neurol Sci. 2016;43(1):15-32.

4. Al-Mufti F, Amuluru K, Damodara N, et al. Novel management strategies for medically-refractory vasospasm following aneurysmal subarachnoid hemorrhage. J Neurol Sci. 2018; 390:44-51.

5. Jaja BNR, Saposnik G, Lingsma HF, et al. Development and validation of outcome prediction models for aneurysmal subarachnoid haemorrhage: the SAHIT multinational cohort study. BMJ. 2018;360:j5745.

6. Shiba M, Ishida F, Miya F, et al. Role of computational fluid dynamics for predicting delayed cerebral ischemia after aneurysmal subarachnoid hemorrhage: study protocol for a multicenter prospective study. Acta Neurochir Suppl. 2020; 127:161-164.

7. Amato A, Britz GW, James ML, et al. An observational pilot study of CSF diversion in subarachnoid haemorrhage. Nurs Crit Care. 2011;16(5):252-260.

8. Maeda Y, Shirao S, Yoneda H, et al. Comparison of lumbar drainage and external ventricular drainage for clearance of subarachnoid clots after Guglielmi detachable coil embolization for aneurysmal subarachnoid hemorrhage. Clin Neurol Neurosurg. 2013;115(7):965-970.

9. Ormond DR, Dressler A, Kim S, Ronecker J, Murali R. Lumbar drains may reduce the need for permanent CSF diversion in spontaneous subarachnoid haemorrhage. Br J Neurosurg. 2013;27(2):171-174.

10. Aydin HE, Ozbek Z, Aydin N, et al. Application of lumbar drainage in vasospasm after spontaneous subarachnoid hemorrhage and prevention of late cerebral infarction. Acta Neurochir Suppl. 2015;120:255-258.

11. Park S, Yang N, Seo E. The effectiveness of lumbar cerebrospinal fluid drainage to reduce the cerebral vasospasm after surgical clipping for aneurysmal subarachnoid hemorrhage. $J$ Korean Neurosurg Soc. 2015;57(3):167-173.

12. Meguro T, Tanabe T, Muraoka K, Terada K, Hirotsune N, Nishino S. Endovascular treatment for aneurysmal subarachnoid hemorrhage with neurogenic pulmonary edema in the acute stage. Turk Neurosurg. 2016;26(6):849-853.

13. Borkar SA, Singh M, Kale SS, et al. Spinal cerebrospinal fluid drainage for prevention of vasospasm in aneurysmal subarachnoid hemorrhage: a prospective, randomized controlled study. Asian J Neurosurg. 2018;13(2):238-246.

14. Liang C, Yang L, Guo S. Serial lumbar puncture reduces cerebrospinal fluid (CSF) infection during removal of hemorrhagic CSF in aneurysmal subarachnoid hemorrhage after endovascular coiling. J Biomed Res. 2018;32(4):305-310.

15. Fang Y, Shao Y, Lu J, et al. The effectiveness of lumbar cerebrospinal fluid drainage in aneurysmal subarachnoid hemorrhage with different bleeding amounts. Neurosurg Rev. 2020;43(2):739-747.

16. Jeong JH, Kim JW, Choi DH. Safety and effectiveness of lumbar cerebrospinal fluid drainage to prevent delayed cerebral ischemia after Fisher grade 3 subarachnoid hemorrhage with minimal intraventricular hemorrhage. Neurochirurgie. 2020;66(4):225-231.

17. Ramakrishna R, Sekhar LN, Ramanathan D, et al. Intraventricular tissue plasminogen activator for the prevention of vasospasm and hydrocephalus after aneurysmal subarachnoid hemorrhage. Neurosurgery. 2010;67(1):110-117.

18. Kai Y, Ito K, Watanabe M, et al. Development of a kit to treat subarachnoid hemorrhage by intrathecal simple urokinase infusion (ITSUKI) therapy: preliminary results in patients with World Federation of Neurological Surgery (WFNS) grade V subarachnoid hemorrhage. World Neurosurg. 2011;75(3-4): 485-490.

19. Etminan N, Beseoglu K, Eicker SO, Turowski B, Steiger HJ, Hänggi D. Prospective, randomized, open-label phase II trial on concomitant intraventricular fibrinolysis and low-frequency rotation after severe subarachnoid hemorrhage. Stroke. 2013;44(8):2162-2168.

20. Litrico S, Almairac F, Gaberel T, et al. Intraventricular fibrinolysis for severe aneurysmal intraventricular hemorrhage: a randomized controlled trial and meta-analysis. Neurosurg Rev. 2013;36(4):523-531.

21. Kramer AH, Roberts DJ, Holodinsky J, et al. Intraventricular tissue plasminogen activator in subarachnoid hemorrhage patients: a prospective, randomized, placebo-controlled pilot trial. Neurocrit Care. 2014;21(2):275-284.

22. Yoshikane T, Miyazaki T, Yasuda S, et al. Aggressive intraoperative cisternal clot removal after clipping aneurismal subarachnoid hemorrhage in elderly patients. World Neurosurg. 2021;147:e482-e490.

23. Webb A, Kolenda J, Martin K, Wright W, Samuels O. The effect of intraventricular administration of nicardipine on mean cerebral blood flow velocity measured by transcranial Doppler in the treatment of vasospasm following aneurysmal subarachnoid hemorrhage. Neurocrit Care. 2010;12(2):159164.

24. Lu N, Jackson D, Luke S, Festic E, Hanel RA, Freeman WD. Intraventricular nicardipine for aneurysmal subarachnoid hemorrhage related vasospasm: assessment of 90 days outcome. Neurocrit Care. 2012;16(3):368-375.

25. Ko SB, Choi HA, Helbok R, et al. Acute effects of intraven- 
tricular nicardipine on cerebral hemodynamics: A preliminary finding. Clin Neurol Neurosurg. 2016;144:48-52.

26. Sadan O, Waddel H, Moore R, et al. Does intrathecal nicardipine for cerebral vasospasm following subarachnoid hemorrhage correlate with reduced delayed cerebral ischemia? A retrospective propensity score-based analysis. J Neurosurg. 2022;136(1):115-124.

27. Barth M, Pena P, Seiz M, et al. Feasibility of intraventricular nicardipine prolonged release implants in patients following aneurysmal subarachnoid haemorrhage. Br J Neurosurg. 2011;25(6):677-683.

28. Carlson AP, Hänggi D, Wong GK, et al. Single-dose intraventricular nimodipine microparticles versus oral nimodipine for aneurysmal subarachnoid hemorrhage. Stroke. 2020;51(4): $1142-1149$.

29. Macdonald RL, Hänggi D, Ko NU, et al. NEWTON-2 Cisternal (Nimodipine Microparticles to Enhance Recovery While Reducing Toxicity After Subarachnoid Hemorrhage): a phase 2 , multicenter, randomized, open-label safety study of intracisternal EG-1962 in aneurysmal subarachnoid hemorrhage. Neurosurgery. 2020;88(1):E13-E26.

30. Sadamasa N, Yoshida K, Narumi O, Chin M, Yamagata S. Milrinone via lumbar subarachnoid catheter for vasospasm after aneurysmal subarachnoid hemorrhage. Neurocrit Care. 2014;21(3):470-475.

31. Ehlert A, Manthei G, Hesselmann V, Mathias K, Bein B, Pluta R. A case of hyperacute onset of vasospasm after aneurysmal subarachnoid hemorrhage and refractory vasospasm treated with intravenous and intraventricular nitric oxide: a mini review. World Neurosurg. 2016;91:673.e11-673.e18.

32. Koyanagi M, Fukuda H, Lo B, et al. Effect of intrathecal milrinone injection via lumbar catheter on delayed cerebral ischemia after aneurysmal subarachnoid hemorrhage. $\mathrm{J} \mathrm{Neu}$ rosurg. 2018;128(3):717-722.

33. Ehlert A, Starekova J, Manthei G, et al. Nitric oxide-based treatment of poor-grade patients after severe aneurysmal subarachnoid hemorrhage. Neurocrit Care. 2020;32(3):742-754.

34. Yamamoto T, Mori K, Esaki T, Nakao Y, Tokugawa J, Watanabe M. Preventive effect of continuous cisternal irrigation with magnesium sulfate solution on angiographic cerebral vasospasms associated with aneurysmal subarachnoid hemorrhages: a randomized controlled trial. J Neurosurg. 2016; 124(1):18-26.

35. Takeuchi S, Kumagai K, Toyooka T, Otani N, Wada K, Mori $\mathrm{K}$. Intravenous hydrogen therapy with intracisternal magnesium sulfate infusion in severe aneurysmal subarachnoid hemorrhage. Stroke. 2021;52(1):20-27.

36. Rzepliński R, Kostyra K, Skadorwa T, Sługocki M, Kostkiewicz B. Acute platelet response to aneurysmal subarachnoid hemorrhage depends on severity and distribution of bleeding: an observational cohort study. Neurosurg Rev. 2021;44(5): 2647-2658.

37. Zimmermann J, Weller J, Grub S, et al. Arginase-1 released into CSF after aneurysmal subarachnoid hemorrhage decreases arginine/ornithine ratio: a novel prognostic biomarker. Transl Stroke Res. Published online February 10, 2021. doi:10.1007/s12975-021-00944-y

38. Nadkarni NA, Maas MB, Batra A, et al. Elevated cerebrospinal fluid protein is associated with unfavorable functional outcome in spontaneous subarachnoid hemorrhage. J Stroke Cerebrovasc Dis. 2020;29(4):104605.

39. Männer A, Thomas D, Wagner M, et al. Sphingosine 1-phosphate levels in cerebrospinal fluid after subarachnoid hemorrhage. Neurol Res Pract. 2020;2(1):49.

40. Vlachogiannis P, Hillered L, Khalil F, Enblad P, RonneEngström E. Interleukin-6 levels in cerebrospinal fluid and plasma in patients with severe spontaneous subarachnoid hemorrhage. World Neurosurg. 2019;122:e612-e618.

41. Qian C, Yu X, Chen J, et al. Effect of the drainage of cerebro- spinal fluid in patients with aneurismal subarachnoid hemorrhage: A meta-analysis. Medicine (Baltimore). 2016;95(41): e5140.

42. Abolfazli E, Fatouraee N, Seddighi AS. Effects of lumbar drainage on CSF dynamics in subarachnoid hemorrhage condition: a computational study. Comput Biol Med. 2016;77: 49-58.

43. Suzuki H, Shiba M, Nakatsuka Y, Nakano F, Nishikawa H. Higher cerebrospinal fluid $\mathrm{pH}$ may contribute to the development of delayed cerebral ischemia after aneurysmal subarachnoid hemorrhage. Transl Stroke Res. 2017;8(2):165-173.

44. Park YK, Yi HJ, Choi KS, Lee YJ, Chun HJ, Kwon SM. Predictive factors of fever after aneurysmal subarachnoid hemorrhage and its impact on delayed cerebral ischemia and clinical outcomes. World Neurosurg. 2018;114:e524-e531.

45. Eicker SO, Beseoglu K, Etminan N, et al. The effect of intraventricular thrombolysis in combination with low-frequency head motion after severe subarachnoid hemorrhage: interim analysis of safety, clot clearance rate and delayed cerebral ischemia. Acta Neurochir Suppl. 2012;114:323-328.

46. Etminan N, Chang HS, Hackenberg K, et al. Worldwide incidence of aneurysmal subarachnoid hemorrhage according to region, time period, blood pressure, and smoking prevalence in the population: a systematic review and meta-analysis. JAMA Neurol. 2019;76(5):588-597.

47. Gaberel T, Gakuba C, Fournel F, et al. FIVHeMA: intraventricular fibrinolysis versus external ventricular drainage alone in aneurysmal subarachnoid hemorrhage: a randomized controlled trial. Neurochirurgie. 2019;65(1):14-19.

48. Garland P, Morton MJ, Haskins W, et al. Haemoglobin causes neuronal damage in vivo which is preventable by haptoglobin. Brain Commun. 2020;2(1):fcz053.

49. Helbok R, Rass V, Kofler M, et al. Intracerebral iron accumulation may be associated with secondary brain injury in patients with poor grade subarachnoid hemorrhage. Neurocrit Care. 2021.

50. Akkaya E, Evran Ş, Çalış F, et al. Effects of intrathecal verapamil on cerebral vasospasm in experimental rat study. World Neurosurg. 2019;127:e1104-e1111.

51. Ehtisham A, Taylor S, Bayless L, Samuels OB, Klein MW, Janzen JM. Use of intrathecal nicardipine for aneurysmal subarachnoid hemorrhage-induced cerebral vasospasm. South Med J. 2009;102(2):150-153.

52. Goodson K, Lapointe M, Monroe T, Chalela JA. Intraventricular nicardipine for refractory cerebral vasospasm after subarachnoid hemorrhage. Neurocrit Care. 2008;8(2):247-252.

53. Kasuya H, Onda H, Takeshita M, Okada Y, Hori T. Efficacy and safety of nicardipine prolonged-release implants for preventing vasospasm in humans. Stroke. 2002;33(4):1011-1015.

54. Kuroi Y, Ohbuchi H, Arai N, et al. Twelve-year single critical care center experience of nicardipine prolonged-release implants in patients with subarachnoid hemorrhage: a propensity score matching analysis. J Neurointerv Surg. 2020;12(8): 774-776.

55. Onal MB, Solmaz I, Civelek E, et al. Comparison of intrathecal dotarizine and nimodipine treatments in cerebral vasospasm after subarachnoid hemorrhage: an experimental study in rabbits. Acta Neurochir Suppl. 2011;110(Pt 2):55-60.

56. Civelek E, Solmaz I, Onal MB, et al. Comparison of intrathecal flunarizine and nimodipine treatments in cerebral vasospasm after experimental subarachnoid hemorrhage in rabbits. Acta Neurochir Suppl. 2011;110(Pt 2):69-73.

57. Mori K, Yamamoto T, Miyazaki M, et al. Effect of intrathecal magnesium sulfate solution injection via a microcatheter in the cisterna magna on cerebral vasospasm in the canine subarachnoid haemorrhage model. Br J Neurosurg. 2012; 26(1):64-68.

58. Onal MB, Bilginer B, Narin F, Ziyal MI, Soylemezoglu F, Ozgen T. Comparison of intrathecal cilostazol and nimodi- 
pine treatments in subarachnoid hemorrhage: an experimental study in rabbits. Acta Neurochir Suppl. 2011;110(Pt 2): 43-48.

59. Fathi AR, Marbacher S, Graupner T, et al. Continuous intrathecal glyceryl trinitrate prevents delayed cerebral vasospasm in the single-SAH rabbit model in vivo. Acta Neurochir (Wien). 2011;153(8):1669-1675.

60. Tangen K, Narasimhan NS, Sierzega K, Preden T, Alaraj A, Linninger AA. Clearance of subarachnoid hemorrhage from the cerebrospinal fluid in computational and in vitro models. Ann Biomed Eng. 2016;44(12):3478-3494.

61. Alotaibi NM, Wang JZ, Pasarikovski CR, et al. Management of raised intracranial pressure in aneurysmal subarachnoid hemorrhage: time for a consensus? Neurosurg Focus. 2017; 43(5):E13.

62. Staykov D, Speck V, Volbers B, et al. Early recognition of lumbar overdrainage by lumboventricular pressure gradient. Neurosurgery. 2011;68(5):1187-1191.

63. Panni P, Donofrio CA, Barzaghi LR, et al. Safety and feasibility of lumbar drainage in the management of poor grade aneurysmal subarachnoid hemorrhage. J Clin Neurosci. 2019; 64:64-70.

64. Reinacher PC, Coenen VA, Kraeutle R, Scheiwe C, Jabbarli R, Roelz R. Feasibility of stereotactic catheter ventriculocisternostomy for cisternal lavage therapy in patients with subarachnoid hemorrhage. Clin Neurol Neurosurg. 2017;163: 94-102.

65. Roelz R, Scheiwe C, Coenen VA, Reinacher PC. A novel rescue therapy for cerebral vasospasm: cisternal nimodipine application via stereotactic catheter ventriculocisternostomy. J Clin Neurosci. 2019;63:244-248.

66. Roelz R, Scheiwe C, Urbach H, Coenen VA, Reinacher P. Stereotactic catheter ventriculocisternostomy for clearance of subarachnoid hemorrhage in patients with coiled aneurysms. Oper Neurosurg (Hagerstown). 2018;14(3):231-235.

67. Christensen ST, Haanes KA, Spray S, et al. Pre-clinical effects of highly potent MEK1/2 inhibitors on rat cerebral vasculature after organ culture and subarachnoid haemorrhage. Clin Sci (Lond). 2019;133(16):1797-1811.

68. Christensen ST, Johansson SE, Radziwon-Balicka A, Warfvinge K, Haanes KA, Edvinsson L. MEK1/2 inhibitor U0126, but not nimodipine, reduces upregulation of cerebrovascular contractile receptors after subarachnoid haemorrhage in rats. PLoS One. 2019;14(4):e0215398.
69. Gatti S, Lonati C, Acerbi F, et al. Protective action of NDPMSH in experimental subarachnoid hemorrhage. Exp Neurol. 2012;234(1):230-238.

70. Gul S, Bahadir B, Hanci V, et al. Effects of ebselen versus nimodipine on cerebral vasospasm subsequent to experimental subarachnoid hemorrhage in rats. J Clin Neurosci. 2010;17(5): 608-611.

71. Solmaz I, Onal MB, Civelek E, et al. The effects of intrathecal nicergoline and nimodipine in cerebral vasospasm: an experimental study in rabbits. Acta Neurochir Suppl. 2011; 110(Pt 2):81-85.

72. Song JN, Zhang M, Li DD, et al. Dynamic expression of the suppressor of cytokine signaling-3 and cytokines in the cerebral basilar artery of rats with subarachnoid hemorrhage, and the effect of acetylcholine. Acta Neurochir (Wien). 2014; 156(5):941-949.

73. Suzuki H, Hasegawa Y, Chen W, Kanamaru K, Zhang JH. Recombinant osteopontin in cerebral vasospasm after subarachnoid hemorrhage. Ann Neurol. 2010;68(5):650-660.

74. Wu CH, Tsai YC, Tsai TH, et al. Valproic acid reduces vasospasm through modulation of Akt phosphorylation and attenuates neuronal apoptosis in subarachnoid hemorrhage rats. Int J Mol Sci. 2021;22(11):5975.

\section{Disclosures}

The authors report no conflict of interest concerning the materials or methods used in this study or the findings specified in this paper.

\section{Author Contributions}

Conception and design: all authors. Acquisition of data: Grossen, Ernst. Analysis and interpretation of data: Grossen, Ernst. Drafting the article: Grossen, Ernst. Critically revising the article: all authors. Reviewed submitted version of manuscript: all authors. Administrative/technical/material support: Grossen. Study supervision: Bauer.

\section{Correspondence}

Audrey A. Grossen: University of Oklahoma Health Sciences Center, Oklahoma City, OK. audrey-grossen@ouhsc.edu. 